\title{
Diffusion-Limited Reactions in Crowded Environments
}

\author{
N. Dorsaz, ${ }^{1}$ C. De Michele, ${ }^{2}$ F. Piazza, ${ }^{3}, *$ P. De Los Rios, ${ }^{3}$ and G. Foffi ${ }^{4}$ \\ ${ }^{1}$ University of Fribourg, Adolphe Merkle Institute, CH-1723 Marly 1, Switzerland \\ ${ }^{2}$ Universita di Roma La Sapienza, Dipartimento di Fisica, P.le Aldo Moro 2, 00185 Roma, Italy \\ ${ }^{3}$ Ecole Polytechnique Fédérale de Lausanne (EPFL), Institute of Theoretical Physics, Laboratory of Statistical Biophysics, \\ 1015 Lausanne, Switzerland \\ ${ }^{4}$ Ecole Polytechnique Fédérale de Lausanne (EPFL), Institute of Theoretical Physics, 1015 Lausanne, Switzerland
} (Received 14 June 2010; published 13 September 2010)

\begin{abstract}
Diffusion-limited reactions are usually described within the Smoluchowski theory, which neglects interparticle interactions. We propose a simple way to incorporate excluded-volume effects building on simulations of hard sphere in the presence of a sink. For large values of the sink-to-particle size ratio $R_{s}$, the measured encounter rate is in good agreement with a simple generalization of the Smoluchowski equation at high densities. Reducing $R_{s}$, the encounter rate is substantially depressed and becomes even nonmonotonic for $R_{s} \ll 1$. Concurrently with the saturation of the rate, stationary density waves set in close to the sink. A mean-field analysis helps to shed light on the subtle link between such ordering and the slowing down of the encounter dynamics. Finally, we show how an infinitesimal amount of nonreacting impurities can equally slow down dramatically the reaction.
\end{abstract}

DOI: 10.1103/PhysRevLett.105.120601

Processes such as gelation, coagulation, crystallization, or self-assembly in colloidal or polymer systems, thinfilm growth in materials science and chemical reactions in biology involve, as a first step, the random movement of particles suspended in a fluid [1]. The components diffuse and when they come into contact, a reaction may be triggered, such as aggregation or assembling. As an example, the usual paradigm for biochemical reactions assumes the formation of an encounter complex, which may subsequently undergo chemical transformation, yielding the product. In most cases, the fixation step proceeds much faster than the encounter, in which case the reaction is said to be diffusion limited. Such reactions are commonly found in biochemical processes such as enzyme catalysis, protein aggregation, and complexation in cells [2].

The simplest model of diffusion-limited encounter in three dimensions has been formulated almost one century ago by Smoluchowski under the hypothesis of noninteracting, spherical, and chemically isotropic reactants, a diffusing particle $(p)$ and a sink (s) [3], leading to the Smoluchowski absorption rate

$$
\kappa_{S}=4 \pi D R \rho_{\infty},
$$

where $\rho_{\infty}$ is the (relative) bulk density of the reactants, $D=D_{s}+D_{p}$ is the coefficient of relative diffusion and $R=R_{s}+R_{p}$ the encounter distance.

The Smoluchowski theory is still nowadays the main theoretical framework within which the aforementioned processes are analyzed. However, this approach is strictly valid only for ideal, infinitely diluted solutions, while most systems become interesting at concentrations far from the ideal gas limit. Cells, for instance, contain a large number of proteins, nucleic acids, and other smaller molecules that
PACS numbers: 05.10.-a, 05.40.Jc, 61.43.Hv, 82.70.Dd

occupy up to $30 \%-40 \%$ of the available volume [4] and that cannot overlap with each other. As a matter of fact, crowding effects are expected to impact profoundly on the thermodynamics and kinetics of biological processes in vivo [4-6], such as protein folding and stability [7] and aggregation [8]. Yet, the effects of crowding on diffusionlimited processes have been examined only in the case of low density of diffusing particles [9] or for tracers diffusing in a medium of inert particles [10-13]. In colloidal science, instead, the properties of crowded environments have been investigated widely. Experiments on concentrated suspensions of nearly hard-core particles revealed unexpected phenomena both in their thermodynamics and in the dynamics, spotlighting a subtle entwining between packing, structuration and dynamics [14].

In this Letter, we generalize the classic Smoluchowski problem to arbitrary crowding conditions by means of a novel computational scheme that allows us to efficiently explore the effects of increasing packing on the encounter dynamics. Typical numerical simulations of diffusionlimited reactions employ different declensions of the Brownian dynamics (BD) algorithm $[15,16]$. Here, we consider a liquid composed of hard spheres of radius $R_{p}$ described through event driven Brownian dynamics (EDBD) [17-19]. This technique allows us to efficiently simulate the stochastic dynamics of many-body hard-core systems. In order to simulate encounter reactions at finite densities, we adapted the EDBD to the configuration of an absorbing sink of radius $R_{s}$ located at the center of a spherical bounding box with constant-flux boundary conditions (Fig. 1) [20].

In this way, by tuning the density in the box, we can measure the encounter rate $\kappa$ and the stationary density profile $\rho(r)$, from the infinite-dilution Smoluchowski limit, 


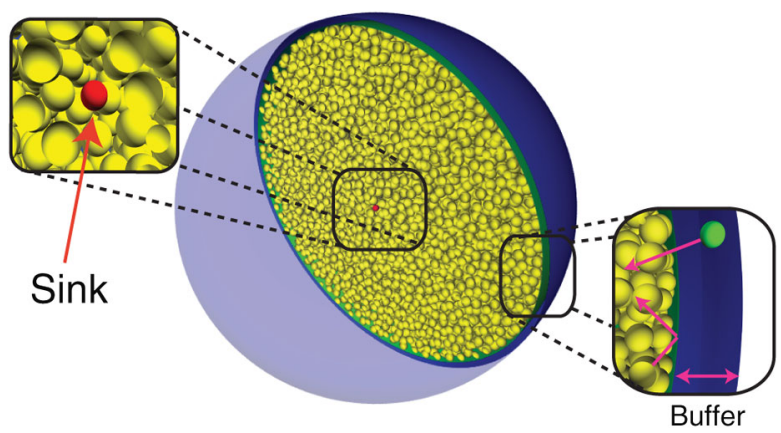

FIG. 1 (color online). Simulation scheme. The reacting particles (light gray or yellow spheres) diffuse in a spherical box. Upon reaching the absorbing sink located at the center of the box, they get absorbed and are reinserted in the external buffer.

namely

$$
\kappa=\kappa_{S}, \quad \rho_{S}(r)=\rho_{\infty}\left[1-\left(R_{s}+R_{p}\right) / r\right]
$$

up to high crowding conditions. In the following, the density is expressed in terms of the corresponding packing fraction of the spheres $\phi$, and lengths in units of $R_{p}$.

As a first investigation of crowding effects, we simulated the case of a large sink-to-particle diameter ratio $\left(R_{s}=\right.$ $8 / 3)$. At low densities $(\phi<0.1)$, the rate increases linearly, in agreement with the $\mathrm{BD}$ and analytical results by Dzubiella and McCammon [9], following the first-order virial correction to the Smoluchowski rate. Increasing the crowding further, the encounter rate is strongly enhanced, reaching even an eightfold magnification at $\phi=0.4$ with respect to the infinitely diluted limit.

In order to account for the strongly nonlinear increase of the rate at intermediate crowding, one can consider the Smoluchowski equation and take interparticle interactions into account in the expression of the net flux $\vec{J}$ through a generalized Stokes-Einstein relation for the collective diffusion coefficient [21]. At first order, crowding effects can be accounted for through the density dependence of the pressure in the bulk liquid $\Pi(\rho)$ (vanishing at zero density), yielding a generalized diffusivity $D(\rho)=D_{0} d[\beta \Pi(\rho)] / d \rho$, where $\beta^{-1}=k_{B} T$ and $D_{0} \equiv \lim _{\rho \rightarrow 0} D(\rho)$. The steady-state Smoluchowski equation in spherical coordinates reads then

$$
\vec{\nabla} \cdot \vec{J}=-\frac{\beta D_{0}}{r^{2}} \frac{d}{d r}\left\{r^{2} \frac{d \rho(r)}{d r} \frac{d \Pi[\rho(r)]}{d \rho(r)}\right\}=0
$$

with the boundary conditions $\rho\left(R_{s}+R_{p}\right)=0$ and $\lim _{r \rightarrow \infty} \rho(r)=\rho_{\infty}$. Integrating the equation that defines the encounter rate, namely $\kappa /\left(4 \pi r^{2}\right)=J(r)$, in the interval $[R,+\infty)$ with $J(r) \equiv|\vec{J}|=\beta D_{0}[d \rho(r) / d r][d \Pi(\rho) / d \rho]$ one obtains

$$
\frac{\kappa}{\kappa_{S}}=\frac{\beta \Pi\left(\rho_{\infty}\right)}{\rho_{\infty}}
$$

where $\beta \Pi\left(\rho_{\infty}\right) / \rho_{\infty}=Z(\phi)$ is the compressibility factor.
The latter can be well approximated through the CarnahanStarling (CS) equation of state $Z(\phi)=\left(1+\phi+\phi^{2}-\right.$ $\left.\phi^{3}\right) /(1-\phi)^{3}$ [22]. At moderate densities, the virial expansion $\beta \Pi(\rho)=\rho\left[1+B_{2} \rho+O\left(\rho^{2}\right)\right]$ yields the Smoluchowski rate at zeroth order and the first-order correction $\kappa / \kappa_{S}=1+B_{2} \rho_{\infty}$ derived in Ref. [9].

For large values of the sink size, the rate increases monotonically with the density in good agreement with Eq. (4), which provides an accurate prediction in the limit $R_{s} \rightarrow \infty$. This is clearly illustrated by inspecting how the rate varies with $R_{s}$ at fixed packing fraction (inset in Fig. 2).

The density gradient between the sink and the bulk regions induces a net force towards the sink, the latter behaving as a depression in a high pressure environment. The rate is thus directly related to the difference in pressure between the diluted region near the sink and the denser bulk. Remarkably, however, when $R_{s}$ is decreased the rate gets substantially depressed at intermediate crowding and becomes even nonmonotonic for $R_{s} \ll 1$, featuring a maximum and a drop at high crowding.

The stationary density distributions of the diffusing particles around the absorbing boundary surface offer a key to understanding the drop in the rate observed at intermediate packing when reducing $R_{s}$. As illustrated in Fig. 3, we found that (i) oscillations set in progressively while increasing $\phi$ and (ii) the structuration is strongly enhanced for small values of $R_{s}$. More specifically, the accumulation of particles around the sink corresponding to the first maximum in the density profiles occurs at a distance $\Delta^{\prime} \simeq\left(R_{s}+R_{p}\right)+\Delta$, where $\Delta$ does not depend on $R_{s}$.

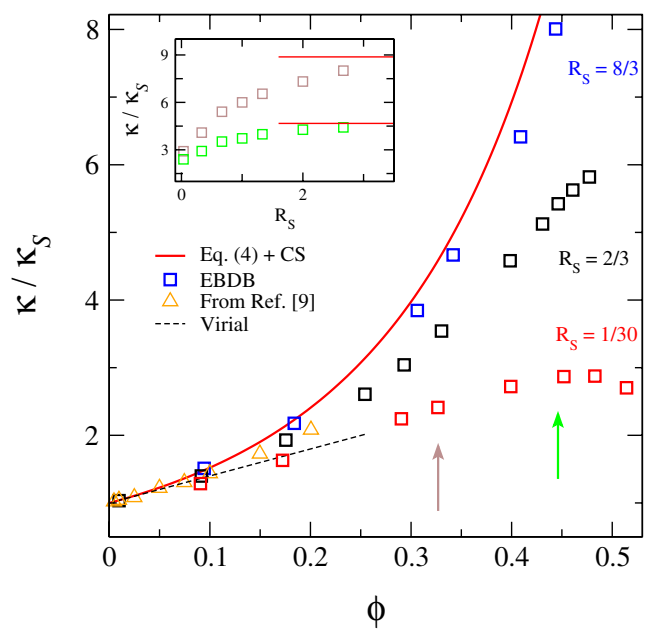

FIG. 2 (color online). Encounter rate versus crowding. For large sink-to-particle diameter ratios $\left(R_{s}=8 / 3\right)$, Eq. (4) accounts for the EDBD results. Reducing $R_{s}$, the rate saturates at high packing, while it displays a maximum for $R_{s} \ll 1$. The virial solution and the BD results of Ref. [9] are also shown. In the inset, we plot the rates versus $R_{s}$ for $\phi=0.33$ and $\phi=0.44$, together with the corresponding theoretical limits predicted by Eq. (4). 
As it is clearly shown in Fig. 3, the progressive structuration of the system bears the clear signature of the radial distribution function $g(r)$ of a hard-sphere liquid at intermediate densities. This effect is by no mean trivial, as $g(r)$ provides a measure of spatial correlations in the reference frame of a moving particle. Here, translational symmetry is broken by the sink, which causes the emergence of a denser layer near the absorber, triggering the onset of stationary density waves.

The simultaneous onset of density oscillations and rate falloff observed upon reducing $R_{S}$ suggests that the organized accumulation of particles is the consequence of a competition among the particles diffusing in the proximity of the sink. In this regard, it is important to note that, $\Delta$ being a constant, the particles compete for the sink at a distance from the absorber that depends on the size of the diffusers only, and not on the size of the latter.

In order to emphasize the connection between the increasing structuration of the liquid and the slowing down of the encounter process it is possible to regard the onset of density fluctuations as marking the appearance of an effective potential between the sink and individual particles. Within such representation, the accumulation of particles at $\Delta$ will act on a freely diffusing particle as an energy barrier $\tilde{V}(r)=f\left(r ; \phi, R_{s}\right)$ [Fig. 4(a)], whose height should depend parametrically on both the bulk concentration $\phi$ and the sink size $R_{s}$ to account for the increasing structuration of the density profile for large values of $\phi$ and small values of $R_{s}$. In general, in the presence of an interaction potential $V(r)$, the Smoluchowski rate reads [23],

$$
\frac{\kappa}{\kappa_{S}}=\left[R \int_{R}^{\infty} \frac{e^{\beta V(r)}}{r^{2}} d r\right]^{-1} .
$$

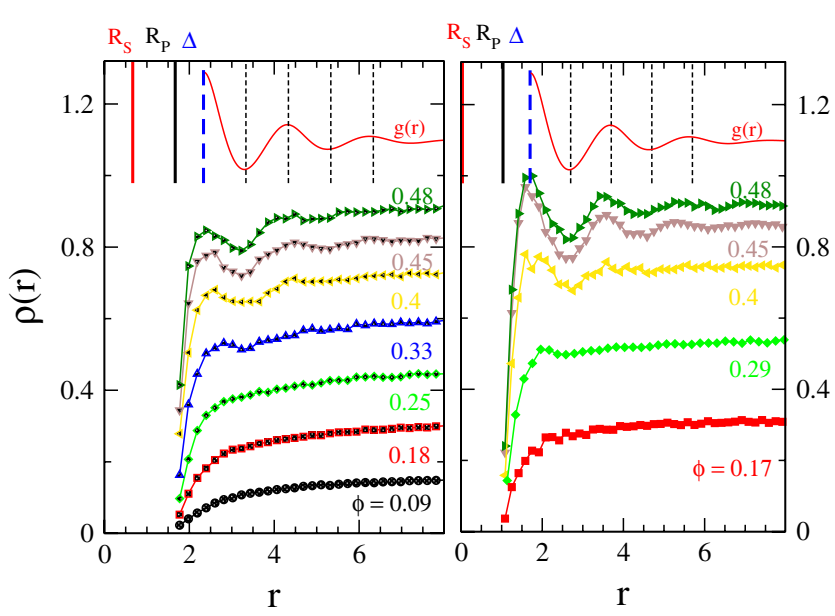

FIG. 3 (color online). Structuration of the density profiles under crowding. The profiles are plotted at increasing bulk packing fraction $\phi$ for $R_{s}=2 / 3$ (left) and $R_{s}=1 / 30$ (right). Structuration is enhanced in the case of the smaller sink, but the liquid gets structured at approximately the same distance $\Delta$ from the contact distance $R_{s}+R_{p}$ in the two cases. The density oscillations are in phase with the radial distribution function $g(r)$ of a hard-sphere liquid at intermediate densities.
It is possible to generalize Eq. (5) in order to account for the effects of crowding by noting that finite densities imply $\kappa \propto \beta \Pi\left(\rho_{\infty}\right)$, as shown by Eq. (4), instead of $\kappa \propto \rho_{\infty}$, as from Eq. (1) (free particles). As a consequence, we may write

$$
\frac{\kappa}{\kappa_{S}}=\frac{\beta \Pi\left(\rho_{\infty}\right)}{\rho_{\infty}}\left[R \int_{R}^{\infty} \frac{e^{\beta \tilde{V}(r)}}{r^{2}} d r\right]^{-1}
$$

It is reasonable to assume that the height $V$ of the potential energy barrier reflects the excess pressure that accumulates in the vicinity of the sink with respect to the ideal case as the packing fraction increases. As a consequence, we assume

$$
\beta \tilde{V}=c \frac{\Pi(\eta)-\Pi_{i d}(\eta)}{\Pi_{i d}(\eta)}=c[Z(\eta)-1],
$$

where $\eta$ is related to the local density at a distance $\Delta$ [Fig. 4(b)] and $\Pi_{i d}(\rho)=\beta \rho$ is the ideal gas pressure. For simplicity, we take $\eta=\rho_{S}(\Delta)$ which introduces the dependence on $R_{s}$ in the effective potential.

Remarkably, a one-parameter fit of Eq. (6) to the numerical data accounts extremely well for both the $\phi$ and $R_{s}$ dependence of the rate [Fig. 4(c)] with a single best-fit value of the floating parameter $(c=3.75)$ for $0<\phi<$ 0.52 and $1 / 30<R_{s}<8 / 3$, thus substantiating our theoretical analysis. Despite its simplicity, this approach seems to capture the essence of the problem, namely, a densityweighted competition among the particles diffusing around the sink. Diffusion-limited reactions in crowded environments are thus the result of a subtle combination of the
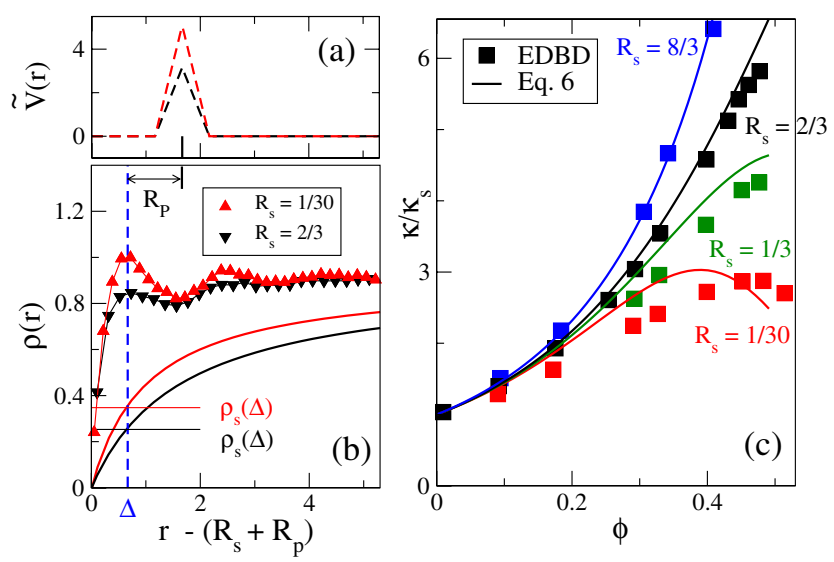

FIG. 4 (color online). Effective-potential description of diffusion-limited reactions under crowding. The effective potential $V\left(\phi, R_{s}\right)$ (a) accounts for the accumulation of particles at distance $\Delta$, resulting in the oscillations in the density profiles (b). The solid lines in panel (b) are plots of the Smoluchowski profiles (1), emphasizing the $R_{s}$ dependence of the density at $\Delta$. (c) The one-parameter fits obtained through Eq. (6) are in excellent agreement with the numeric results for all values of $R_{s}$, providing a quantitative link between the drop in the rate and the onset of stationary density oscillations. 


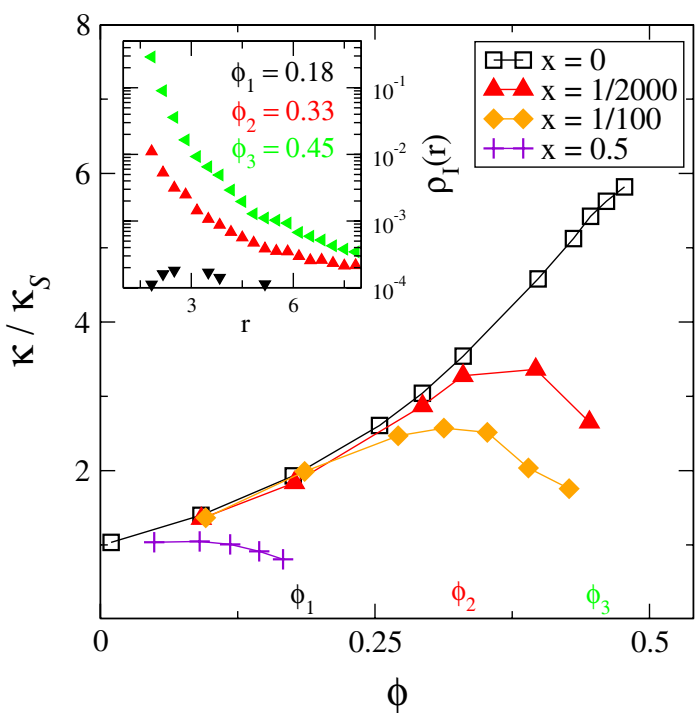

FIG. 5 (color online). Encounter rate versus fraction of nonreacting impurities $x$ for $R_{s}=2 / 3$. Inset: the density profile for impurities $\phi_{I}(r)$ for $x=1 / 2000$.

liquid properties of the diffusers with the underlying longrange Smoluchowski description.

As a first extension of our numerical scheme to more complicated diffusion-limited reactions [4,8,24], we investigated the effects of introducing a finite amount of nonreacting hard-sphere impurities $(I)$ in the system. We found that an infinitesimal fraction $(x=1 / 2000)$ is sufficient to slow down considerably the reaction with respect to the one-component case (Fig. 5). While at low crowding the rate and the density profiles are insensitive to the presence of nonreacting particles, an accumulation of the latter against the sink takes place when increasing $\phi$ above 0.35. As a result, the sink gets screened to the reacting particles and the rate falls off. Indeed, when $\phi$ is increased, the impurities get trapped at the sink surface because of the large density gradient, and need to overcome a substantial energy barrier in order to get back to the bulk region [25]. The extreme sensitivity of the reaction rate to infinitesimal amounts of impurities might, for instance, be of great interest in the context of molecular trafficking across the nuclear pore complex, a protein complex responsible for protected exchange between the nucleus and the cytoplasm, or for preventing the transport of undesirable material through the nuclear envelope [26].

In conclusion, in this Letter we tackled a fundamental open issue. Our novel approach clearly highlights the physical ingredients necessary for the derivation of a self-consistent Smoluchowski theory valid for crowded environments. Moreover, while jamming phenomena like arches at bottleneck or at escape issues are well known in granular media or traffic studies [27], this is, to the best of our knowledge, the first time that a complex ordering in the presence of an absorbing boundary is reported for a system that is still in a liquidlike state.

N.D. acknowledges support from the Adolphe Merkle Foundation, C.D.M. by ERC (226207PATCHYCOLLOIDS), SNSF (visiting Grant No. IZK022-121268). G. F. acknowledges support by the SNSF (Grant No. PP0022_119006).

*Present address: Centre de Biophysique Moléculaire (CBM-CNRS), Rue Charles Sadron, 45071 Cedex 2 Orléans, France.

[1] F. Family and D. P. Landau Kinetics of Aggregation and Gelation (North-Holland, The Netherlands, 1984).

[2] S. A. Rice, Diffusion-Limited Reactions (Elsevier, New York, 1985).

[3] M. V. Smoluchowski, Z. Phys. Chem. 92, 129 (1917).

[4] R. J. Ellis and A.P. Minton, Nature (London) 425, 27 (2003).

[5] T. A. Ryan et al., Science 239, 61 (1988).

[6] S. B. Zimmerman and A. P. Minton, Annu. Rev. Biophys. Biomol. Struct. 22, 27 (1993).

[7] M. S. Cheung, D. Klimov, and D. Thirumalai, Proc. Natl. Acad. Sci. U.S.A. 102, 4753 (2005).

[8] A. P. Minton, Curr. Opin. Struct. Biol. 10, 34 (2000).

[9] J. Dzubiella and J. A. McCammon, J. Chem. Phys. 122, 184902 (2005).

[10] W. Dong, F. Baros, and J. C. Andre, J. Chem. Phys. 91, 4643 (1989).

[11] J. Sun and H. Weinstein, J. Chem. Phys. 127, 155105 (2007).

[12] J. D. Schmit, E. Kamber, and J. Kondev, Phys. Rev. Lett. 102, 218302 (2009).

[13] J. S. Kim and A. Yethiraj, Biophys. J. 98, 951 (2010).

[14] P. N. Pusey and W. van Megen, Nature (London) 320, 340 (1986).

[15] D. L. Ermak and J. A. McCammon, J. Chem. Phys. 69, 1352 (1978).

[16] B. Cichocki and K. Hinsen, Physica (Amsterdam) 187A, 133 (1992).

[17] G. Foffi et al., Phys. Rev. Lett. 94, 078301 (2005).

[18] A. Scala, T. Voigtmann and C. De Michele, J. Chem. Phys. 126, 134109 (2007).

[19] C. De Michele J. Comput. Phys. 229, 3276 (2010).

[20] N. Dorsaz et al., J. Phys. Condens. Matter 22, 104116 (2010).

[21] J. Dhont, An Introduction to Dynamics of Colloids (Elsevier, Amsterdam, 1996).

[22] N. Carnahan and K. Starling, J. Chem. Phys. 51, 635 (1969).

[23] P. L. Houston, Chemical Kinetics and Reaction Dynamics (Dover Publications, New York, 1985).

[24] A. Zaccone et al., Phys. Rev. E 80, 051404 (2009).

[25] Details of the simulations reported in a forthcoming study.

[26] F. Alber et al., Nature (London) 450, 695 (2007).

[27] D. Helbing, I. Farkas, and T. Vicsek, Nature (London) 407, 487 (2000). 